\title{
Experimental Evaluation of an Accelerating Lens on Spatial Field Structure and Frequency Spectrum
}

\author{
Astapenya, V.a, Sokolov, V.a , Ageyev, D. b \\ aBorys Grinchenko Kyiv University, Kyiv, Ukraine \\ ${ }^{\mathrm{b}}$ Kharkiv National University of Radio Electronics, Kharkiv, Ukraine
}

\begin{abstract}
The results of experimental studies of the effect of an accelerating metal plate lens used to increase the signal level in the direction of the remote subscriber of the wireless access system on the frequency spectrum of the received signal, the field structure in the horizontal and vertical plane, and polarization are presented. An insignificant (up to $20 \mathrm{kHz}$ ) narrowing of the spectrum of the radio signal of the Wi-Fi system using a lens was revealed. The effect of the lens on the field distribution in the azimuthal plane is clarified. The angular parameters of the shading areas are estimated where the reception conditions worsen when the lens is located near the access point. In the vertical plane, the lens significantly narrows the angular sector of the radiation. The measurements were carried out under conditions typical of such systems. A brief description of the measuring complex developed for these studies is given. (C) 2020 IEEE.
\end{abstract}

\section{Author keywords}

Accelerating Metal Plate Cylindrical Lens, Field Distribution, Phase Speed, Remote Subscriber, Signal Frequency Spectrum, Wireless Access Point

About this paper

https://ieeexplore.ieee.org/document/9252755/

Online ISBN: 978-172817313-9

DOI: $10.1109 /$ UkrMW49653.2020.9252755

EID: $\underline{2-S 2.0-85097710865}$

First Online: 11 November 2020

Original language: English

Publisher: IEEE Inc. 